\title{
An Intelligent Sales Assistant for Configurable Products
}

\author{
Martin Molina \\ Department of Artificial Intelligence, Technical University of Madrid \\ Campus de Montegancedo s/n, 28660 Boadilla del Monte (Madrid), Spain \\ Email: mmolina@fi.upm.es
}

\begin{abstract}
Some of the recent proposals of web-based applications are oriented to provide advanced search services through virtual shops. Within this context, this paper proposes an advanced type of software application that simulates how a sales assistant dialogues with a consumer to dynamically configure a product according to particular needs. The paper presents the general knowledge model that uses artificial intelligence and knowledge-based techniques to simulate the configuration process. Finally, the paper illustrates the description with an example of an application in the field of photography equipment.
\end{abstract}

\section{Introduction}

Some of the recent proposals in e-commerce have been oriented to improve the communication and the level of assistance to the consumer by providing more active webbased applications including semantic features. For this purpose, advanced techniques from different fields such as artificial intelligence (knowledge-based systems, natural language, etc.) and advanced user-system interaction (multimedia presentations, 3D graphics, etc.) are being applied [1]. For example, Ebrain uses rule-based knowledge representation and natural language techniques to guide visitors through a website explaining products and special offers. Another example is given by [2] that proposes virtual shopping advisor that makes recommendations after a cluster-based analysis of the customer.

To provide this type of support, these applications need to automatically interpret diverse and detailed knowledge about the products and their context. This is especially important in the case of markets of configurable products, i.e., products that need to be dynamically configured according to the needs of customers by assembly specific components. The complexity and diversity of knowledge for this type of applications requires appropriate technical solutions. In this context, this paper contributes with a general approach to this problem. The paper presents how this type of application can be supported by knowledge-based architecture using problem-solving methods from artificial intelligence and, the paper illustrates the description with an application that was developed in the field of photography equipment. 


\section{The knowledge model for the sales assistant}

In order to develop the web-based application, we followed the general idea of intelligent assistant [3], a type of software application oriented to help operators in making decisions. We followed this approach to design a system, conceived as a virtual sales assistant, with the following characteristics: (1) the system recommends candidate product configurations based on the interpretation of the customer needs, (2) the systems tries to justify the proposals with convincing explanations, and (3) the customer must be able of changing (total or partially) the proposals.

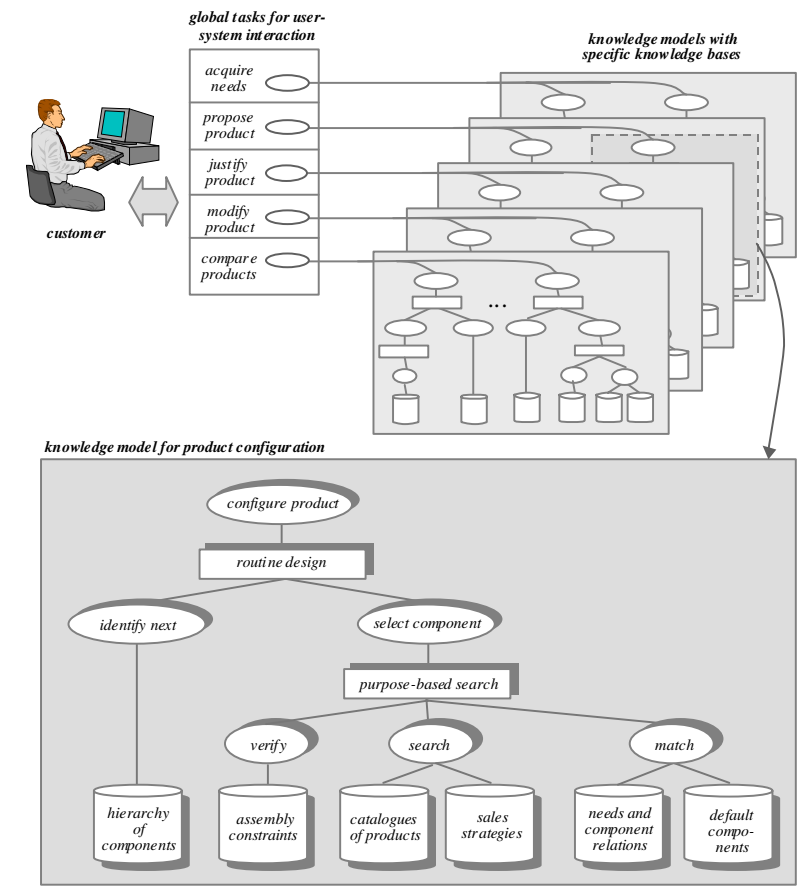

Figure 1: General view of the knowledge organization for the intelligent sales assistant [4]

To provide this support the sales assistant must bring together different types of knowledge: (1) knowledge about the products, (2) knowledge about the customer, and (3) knowledge about the company interests. We designed a computer-based solution using knowledge-based techniques, following the recent trends in knowledge engineering techniques that apply a model-based view (CommonKADS [5] or Protégé-2000 [6]). Figure 3 shows a general view of the knowledge organization. The figure shows the main global tasks, which support the interaction with the customer, associated to the corresponding knowledge models. Details about this model can be found at [4]. 


\section{Symbolic knowledge representation}

The previous model includes a number of types of knowledge bases that, for instance, in a particular domain with 10 generic components, would produce a total of 37 specific knowledge bases. The particular software implementation requires to associate a particular symbolic representation and inference procedure for each knowledge base and to establish the corresponding control mechanisms for the execution of the global model. For this purpose, advanced knowledge engineering tools can be used such as the KSM environment [7], [8]. For example, the knowledge about relations between customer-needs and components, and the knowledge about assembly constraints can be formulated by a particular frame-based representation (figure 3) with a special matching inference procedure. Each frame represents a type of component (e.g., a color CRT monitor, LCD monitor, etc.) and each slot of the frame can be either a customer need (e.g., type-of-image: color-medium-quality) or an assembly constraint. In addition to that, explicit logical expressions formulate control knowledge for frame matching. Figure 2 shows an example of search tree developed during the configuration process. Each ellipse represents a task-execution that develops additional inference processes in local search spaces using particular knowledge bases. In addition to this, the system includes other inference processes for different functions (justification, comparison, etc.) using other knowledge bases with different representation formalisms (hierarchies, rules, logic, etc.).

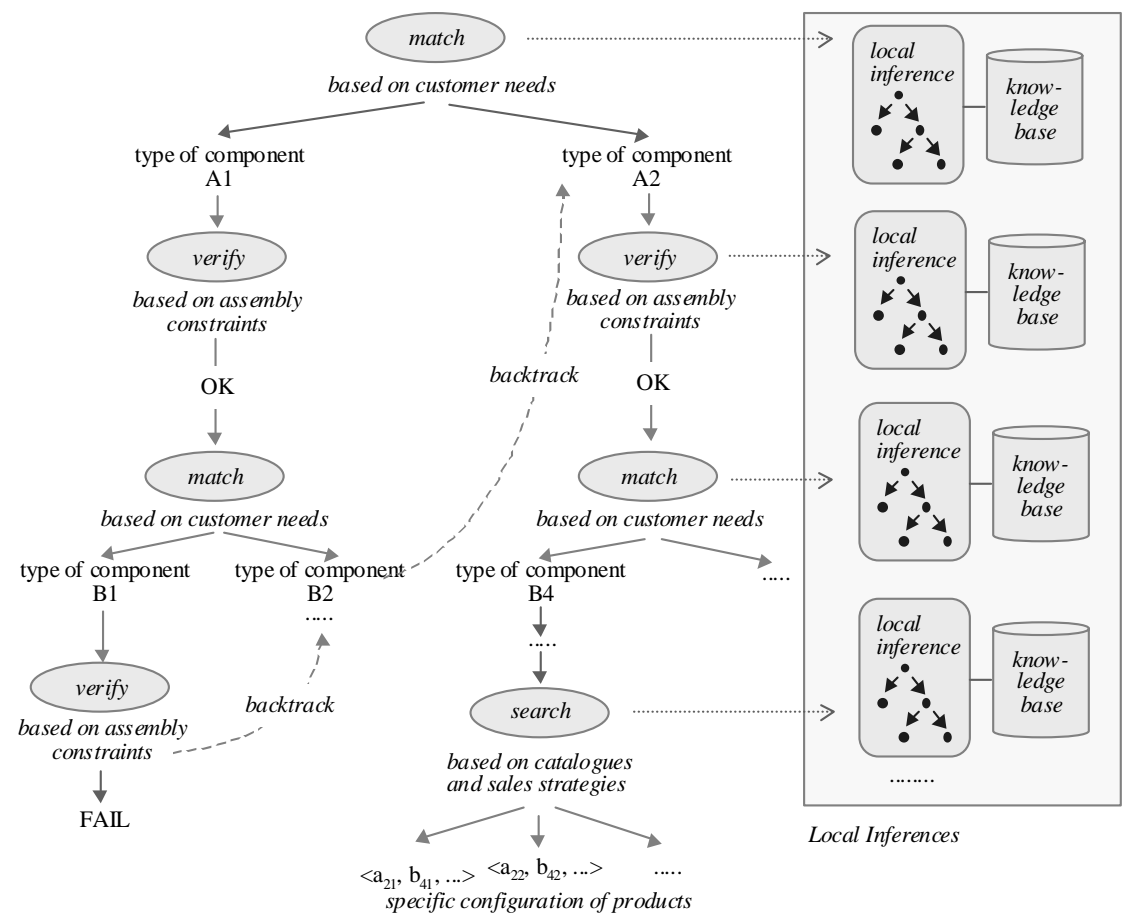

Figure 2: Example of search tree developed during the configuration process. 


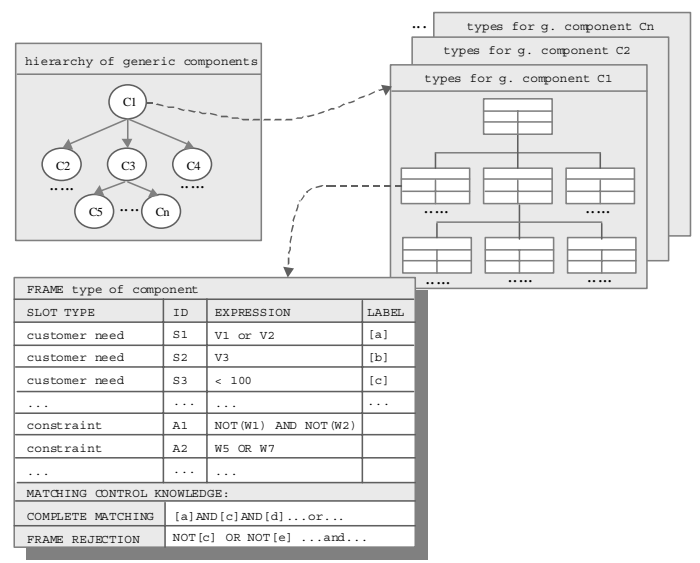

Figure 3: Example of part of the symbolic representation for the intelligent sales assistant.

\section{Application}

Following this general approach, a system was developed for a real-world application in the field of photography equipment. A website prototype was developed to assist potential customers in buying equipment of photography. Figure 4 shows an example of some windows presented by the application in the website (Spanish language). In this case, according to the partial data about the customer, the assistant proposes an initial solution based on a compact camera and additional complements. The customer can ask for justification about this proposal and the assistant provides explanations based on the needs that were satisfied. The customer can also change the proposal asking for alternative products. In all these cases, the configuration process is repeated considering the new needs and constraints. In addition to that, the customer can express its level of satisfaction. With this information the system adapts the user preferences in order to propose more adequate answers.

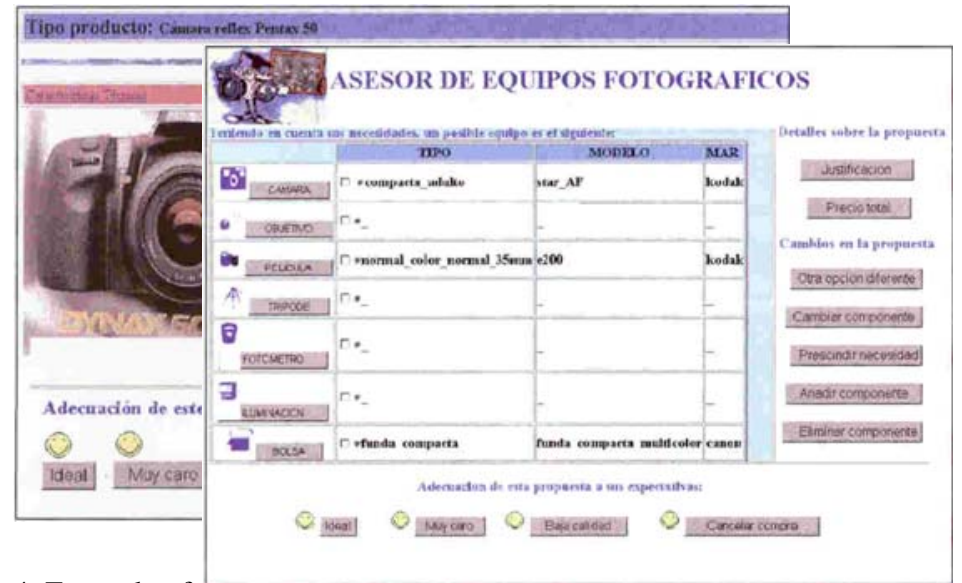

Figure 4: Example of windows of the web-based application for photography equipment. 


\section{Conclusions}

In summary, the proposal presented in this paper constitutes an innovative solution to develop a particular type of web-based application. The paper describes a solution to simulate the behavior of a sales assistant for configurable products. For this purpose, artificial intelligence and knowledge-based techniques are applied to explicitly represent and automatically interpret detailed knowledge about products, customers and the company strategies. The model-based approach, recently followed in the field of knowledge engineering, has been appropriate to formulate the different types of knowledge and the particular flexible and efficient operational implementation. The model described in the paper has been validated with the development of an application in the field of photography equipment. This experience has opened new possibilities for the development of future projects in different domains.

Acknowledgements. This work was developed in the Intelligent Systems Research Group (Dep. Artificial Intelligence, Tech. Univ. Madrid). Rocío Serna was in charge of the development of the web-based application for photography equipment.

\section{References}

1. Terpsidis I., Moukas A., Pergioudakis B., Doukidis G., Maes P.: "The Potential of Electronic Commerce in Re-engineering Consumer-Retail Relationships through Intelligent Agents”. Roger J.Y. et al (Eds.). Advances in Information Technologies: The Business Challenge, IOS Press, 1997.

2. Urban G.L., Sultan F., Qualls W.: "Placing Trust at the Center of Your Internet Strategy" Sloan-Management-Review. vol. 42, No.1, pp. 39-48. ISSN 0019-848X, 2000.

3. Boy, G., Gruber T.R.: "Intelligent Assistant Systems: Support for Integrated HumanMachine Systems” Proceedings of 1990 AAAI Spring Symposium on Knowledge-Based Human-Computer Communication, March 1990, Stanford University.

4. Molina M. "Modeling Commercial Knowledge to Develop Advanced Agent-based Marketplaces for E-commerce” Proc. of the Fifth International Workshop CIA-2001 on Cooperative Information Agents Modena (Italy). Lecture Notes in Artificial Intelligence series, Springer. 2001.

5. Schreiber G., Akkermans H., Anjewierden A., De Hoog R., Shadbolt N., Van de Velde W., Wielinga B.: "Knowledge engineering and management. The CommonKADS methodology” MIT Press, 2000.

6. W.E. Grosso, H. Eriksson, R. W. Fergerson, J. H. Gennari, S. W. Tu, \& M. A. Musen: "Knowledge Modeling at the Millennium (The Design and Evolution of Protege-2000)". Technical Paper No. SMI-1999-0801. Stanford Medical Informatics. Stanford University

7. Cuena J., Molina M.: "The role of knowledge modelling techniques in software development: a general approach based on a knowledge management tool” International Journal of Human-Computer Studies. No. 52. pp 385-421. Academic Press, 2000.

8. Molina M., Sierra J.L., Cuena J.: "Reusable Knowledge-based Components for Building Software Applications: A Knowledge Modelling Approach” International Journal of Software Engineering and Knowledge Engineering. vol 9, No. 3, pp 297-317., 1999. 